\title{
Kurse und Kongresse in Ungarn
}

Auch dieses Jahr werden wieder zahlreiche SGUM-anerkannte Weiter- und Fortbildungskurse, vornehmlich in Sonografie des Bewegungsapparates, in Ungarn, aber in deutscher Sprache durchgeführt. Neu und bereits mehrfach erfolgreich absolviert, sind sog. Hospitationskurse im Angebot. In Kleinstgruppen von nur 2-3 Teilnehmern wird an zweieinhalb Tagen intensiv praktisch gegenseitig und an vielen Patienten geübt. Daneben kommen aber die lokale Gastronomie, Kultur und Erholung auch zum Zug.

Folgende SGUM-anerkannte Weiter- und Fortbildungskurse sind in diesem Jahr noch ausgeschrieben:
Kurse in Sonografie des Bewegungsapparates

- Grundkurs 3.-6. Juli 2014

- Aufbaukurs 12.-15. Juni 2014

- Schlusskurs 4.-7. Sept. 2014

\section{Hospitationskurse}

- 4/2014 15.-18. Mai 2014

- 5/2014 10.-13. Juli 2014

- 6/2014 18.-21. Sept. 2014

Weitere Hospitationskurse sind noch vorgesehen und werden später publiziert. Alle Kurse sind auch für Teilnehmer aus den Nachbarländern offen. Auch Begleitpersonen können mitgebracht werden.
Vom 3.-9. August können auch „Sono-Ferien“ für Teilnehmer mit Partner oder Familie gebucht werden mit praktischen Übungen (analog „Sonohöhle“) nach individuellem Wunsch.

Alle Einzelheiten und das Anmeldeformular sind auf www.ungarnkurse.info aufgeschaltet.

\section{Kongresse}

An dieser Stelle sei auf den folgenden Kongress hingewiesen:

- 12th Biannual Congress of the „International Society for Musculoskelettal Ultrasound (ISMUS)“

- 16. - 18. Oktober 2014, Novotel Szeged (Ungarn)

Mit Vorkursen in Hüftsonografie und Sonografie des Bewegungsapparates

Einzelheiten und Anmeldung unter www. ismus-congress.info 